\title{
The Perceived Needs Questionnaire for Dementia Informal Caregivers (PNQ-DIC): Development and initial validation
}

Tânia Brandão ${ }^{1,2}$, Rute Brites ${ }^{1}$, Francisco Moniz Pereira ${ }^{3}$, João Hipólito ${ }^{2}$, \& Odete Nunes ${ }^{1}$ ${ }^{1}$ CIP, Department of Psychology, Autonomous University of Lisbon, 1150-023 Lisbon, Portugal

${ }^{2}$ CPUP - Center for Psychology at University of Porto

${ }^{3}$ Lisbon Psychiatric Hospital, Lisbon, Portugal

This research has been partially funded by Portuguese national funds through FCT -

Portuguese Foundation for Science and Technology - as part of the project Centre for Research in Psychology - UAL Ref ${ }^{a}$ UID/PSI/04345/2019. Correspondence concerning this paper should be addressed to Tânia Brandão, CIP, Department of Psychology, Autonomous University of Lisbon, 1150-023 Lisbon, Portugal. Email: tbrandao@autonoma.pt

Please Note: This is the author's version of the manuscript accepted for publication in Psychogeriatrics. Changes resulting from the publishing process, namely editorial editing, corrections, final formatting for publication, and other modifications resulting from quality control mechanisms may have been subsequently added.

A definitive version was subsequently published as: Brandão, T., Brites, R., Pereira, F. M., Hipólito, J., \& Nunes, O (2020). The Perceived Needs Questionnaire for Dementia Informal Caregivers (PNQ-DIC): Development and initial validation. Psychogeriatrics. Doi: 10.1111/PSYG.12512 


\section{The Perceived Needs Questionnaire for Informal Dementia Caregivers: Development and initial validation}

Despite the recognition of the value of informal caregiving, the recognition of its burden, and the recognition of the prevalence of unmet needs, little consensus exist about what are the unmet needs of dementia informal caregivers and they should be measured within this context ${ }^{1}$. The assessment/recognition of (unmet) needs in informal caregiving in the context of dementia should be a priority given its potential role on the design and development of services, policies, and psychosocial interventions capable of alleviating caregivers' burden. While many instruments exist to assess informal caregivers needs (see for a review ${ }^{2}$ ), none of them was developed and validated to account for the specific needs of informal dementia caregiving. In this study, we present the process used to develop and evaluate the psychometric properties of a novel and rapid self-report instrument for both research and clinical use designed to measure the needs of informal dementia caregivers - the PNQ-IDC.

The sample was composed of 71 caregivers of patients with dementia ( 47 females; $65 \%$; Mage $=64.87 ; S D=13.99)$. Participants characteristics are presented in supporting information (Table S1). The present study was approved by the Ethical Committee $\|$ removed for blind review $\|$. Caregivers were recruited at two public hospitals and one health care center in Lisbon. A written informed consent was obtained from all participants. A deductive method was used to item generation. We investigated relevant literature and generated 14 items based on coauthors clinical experience. The generation of items were made taking into account the identification of needs that could be supplied with adequate support provided by their health care professionals. Participants were asked to respond to the items using a 5-point Likert scale ranging from 1 (nothing) to 5 (very much). They were instructed to identify the additional needs they have (beside those that are already being supplied) which means that nothing answers do 
mean that there is no need but that this need does not require an intervention at the moment. In order to examine concurrent validity, caregivers' subjective burden was assessed using the Zarit Burden Interview (Portuguese version ${ }^{3}$ ). This measure was applied to the same participants. The Cronbach's alpha for this study was .88 .

Descriptive statistics are presented in supporting information (Table S2). Exploratory factor analysis (EFA) was carried out using principal components analysis (PCA) and varimax rotation $^{4}$ was performed because the potentially extracted factors were presumed to be uncorrelated. The Kaiser-Meyer-Olkin $(\mathrm{KMO})$ value $(\mathrm{KMO}=.74)$ and the significance of the Bartlett's test of sphericity $(p<.001)$ evidenced that data was suitable for factor analysis. Factor solutions were determined by the results of the parallel analysis (Parallel Analysis Engine ${ }^{5}$, by the inspection of the scree plot $^{4}$ and by the amount of the proportion of variance accounted for and by the interpretability of the factors ${ }^{4}$. Criteria suggested three and four components. These two alternative factor solutions were tested. The four-factor structure revealed difficult to interpret, with two components that seemed to overlap in its content (namely, home care nursing and equipment needs, plus human resources and functional needs). The three-factor solution achieved the best theoretical interpretability (see Table 1) and accounted for $64 \%$ of variance. One item presented cross loadings and for that reason was excluded (item 1). The factor 1 was labeled "informational and practical needs", was composed by items $6,7,8,9$, and 10 and refers to the information and practical needed in terms of the disease, treatments and symptoms management; the factor 2 was labeled "human resources and functional needs", was composed by items 2, 3, 4, and 5 and refers to dimensions of structures and health care professionals; finally, the factor 3 was labeled "psychosocial needs" and was composed by items 11 and 12 and refers to mental needs and desired support. Internal reliability and intercorrelations between factors are presented in supporting information (Table S3). The Cronbach's alphas were good ranging from .73 to .89; the low to 
moderate intercorrelations between factors evidenced the multidimensionality of the scale. A significant positive correlation was found between psychosocial needs and subjective burden.

Our findings suggest that the PNQ-IDC seems to be feasible given the lack of missing data as well as the lack of negative comments about items. Moreover, preliminary results suggest that this instrument is valid and reliable to assess unmet needs. It has the advantage of being a short instrument and, for this reason, it can be applied both for clinical and research purposes without increasing the burden of participants.

\section{Acknowledgments/Funding:}

This research has been partially funded by Portuguese national funds through FCT - Portuguese Foundation for Science and Technology - as part of the project Centre for Research in Psychology - UAL Ref UID/PSI/04345/2019.

\section{Disclosure statement:}

The authors have no potential conflicts of interest to disclose.

\section{References}

1- Bangerter LR, Griffin JM, Zarit SH, Havyer R. Measuring the needs of family caregivers of people with dementia. An assessment of current methodological strategies and key recommendations. J Appl Gerontol. 2017. doi: 10.1177/0733464817705959.

2 - Lefranc A, Pérol D, Plantier M, Chatelain P, de Rohan-Chabot H, Schell M. Assessment of informal caregiver's needs by self-administered instruments: a literature review. Eur. J. Public Health. 2017;27:796-801.

3 - Pereira MG, Sobral M. Entrevista de Zarit de sobrecarga do Cuidador. In A. Mendonça, \& M. Guerreiro (Coord). Escalas e Testes na Demência (2a ed). Porto: Grupo de Estudos de Envelhecimento Cerebral e Demência: 2007. 
4 - Tabachnick BG, Fidell LS. Using multivariate statistics. Allyn \& Bacon/Pearson Education: 2014.

5 - Patil VH, Singh SN, Mishra S, Donavan DT. Parallel analysis engine to aid determining number of factors to retain [Computer software]. Verfügbar Unter: 2007.

\section{Supporting information:}

Additional supporting information may be found in the online version of this article at the publisher's website

Table S1 - Participants Characteristics

Table S2 - Descriptive and Item Analyses of the PNQ-DIC

Table S3 - Internal consistency and intercorrelations among PNQ-ICD subscales 